Christiane Alpers*

\title{
Ontologische Erlösung und menschliche Sünde: John Milbank und Kathryn Tanner über die Erlösung der Menschheit durch die exzessive Positivität Gottes
}

DOI 10.1515/nzsth-2016-0012

Zusammenfassung: In diesem Artikel untersuche ich die Rolle der menschlichen Sündhaftigkeit in John Milbanks Erlösungsverständnis. Milbank argumentiert, dass eine wahrhaft gedeihende Kultur nicht auf säkulare Philosophien, sondern auf eine christliche Theologie aufbauen müsse. Um gegenwärtige gesellschaftliche Probleme zu überwinden, muss die Welt erneut in ihrer Partizipation an Gott gedacht werden. Es wurde vermehrt kritisiert, dass diese Behauptung vernachlässige, dass jeder Mensch von der Sünde erlöst werden muss, bevor solch eine Partizipation Wirklichkeit ist. Nach einer kurzen Darstellung dieses Kritikpunkts verteidige ich Milbank im zweiten Teil dieses Artikels, indem ich argumentiere, dass Milbank das Spannungsverhältnis zwischen Erlösung und Sünde im Sinne der exzessiven Gutheit der Wirklichkeit und des Mangels an Wirklichkeit der Sünde versteht. Die Erlösungswirklichkeit wird durch jeden zusätzlichen erlösten Menschen erweitert. Menschliche Sündhaftigkeit hat auf diese Wirklichkeit keinen Einfluss, da Sünden im Nichts verschwinden. Zuletzt versöhne ich diese Erlösungsvorstellung mit den Einwürfen seitens Milbanks Kritiker / innen, indem ich mich an Kathryn Tanners Theologie wende, die mit Milbank bezüglich der unumstößlichen Erlösungswirklichkeit übereinstimmt. Jedoch weist sie größere Sensibilität für die bei Milbank beanstandete Marginalisierung der Wichtigkeit der Sündenlehre auf. Tanner schlägt vor, dass die exzessive Gutheit der Wirklichkeit menschliche Gutheit dermaßen übersteigt, dass die Partizipation der Welt an Gott erstrangig ein unnachlässiges Vergeben der menschlichen Sündhaftigkeit und nur in äußerst seltenen Fällen eine Erweiterung des wahrhaft Guten durch menschliche Gutheit darstellt. Dies führt zu einer bescheideneren christlichen Kultur als diejenige, welche Milbank sich vorstellt.

Schlüsselwörter: Radical Orthodoxy, Erlösung, Partizipation, Sünde, christliche Gesellschaftskritik

*Korrespondenzautor: Christiane Alpers, Faculty of Philosophy, Theology and Religious Studies, Radboud University, Erasmusplein 1, Postbus 9103, 6500 HD Nijmegen, Netherlands,

E-Mail: c.alpers@ftr.ru.nl 
Summary: This article examines the role of human sinfulness in John Milbank's understanding of redemption. John Milbank argues that Christian theology, instead of secular philosophies, must be the basis of a truly flourishing culture. The world must be re-imagined as participating in God in order to overcome current societal problems. This claim has been criticized for neglecting that every human being must be redeemed from sin before the world's participation in God is real. After a brief introduction of this criticism, I defend Milbank in the second part of this article by way of arguing that he construes the tension between redemption and human sinfulness in terms of the excessive goodness of reality and sin's lack of reality. Redemption is expanded through the addition of every redeemed human being. Sinfulness cannot alter this reality because sin merely slides into nothingness. Finally, I reconcile this vision with Milbank's critics by drawing on insights from Kathryn Tanner who is in agreement with Milbank about the unquestionable reality of redemption but respects more Milbank's critics' sensibility for the seriousness of sin. She suggests that the excessive goodness of redeemed reality surpasses ordinary human goodness to such an extent that the world's participation in God means primarily a continuous overcoming of human sinfulness and only in exceptional cases an expansion of the really Good through human goodness. This leads to a more humble cultural project than Milbank's own.

Keywords: Radical Orthodoxy, Redemption, Participation, Sin, Christian Social Criticism

\section{Einleitung}

John Milbank ist vor allem im angelsächsischen Raum für seine provokante These bekannt, dass eine christliche Ontologie sich besser für die Organisation der Gesamtgesellschaft eignet als ihr säkulares Gegenstück. Für die Situation westlicher Gegenwartsgesellschaften bedeutet dies, dass die Welt erneut in ihrer Beziehung zu Gott gedacht werden muss, um derzeitige politische Probleme zu überwinden. Die Qualität und Standfestigkeit dieser These wird noch stets sehr unterschiedlich bewertet. In den letzten Jahren hat John Milbanks Werk zunehmend Anklang bei protestantischen Theolog/innen gefunden. Während der Versuch einer christlichen Gesellschaftskritik im Allgemeinen meist Wohlwollen findet, werden jedoch bestimmte Aspekte von Milbanks Christologie wiederholt kritisiert. Dieser Artikel bezieht sich auf die Behauptung, dass Milbanks triumphalistisches Rühmen einer christlichen Ontologie über ihren häretischen säkularen Gegenspieler auf Kosten adäquaterer dogmatischer Überlegungen erworben 
wird. Insbesondere widme ich mich dem Vorwurf, Milbank bagatellisiere die Rolle der Sündenlehre. Während er postuliert, gegenwärtige gesellschaftliche Probleme durch die Wiederbelebung einer analogen Weltsicht lösen zu können, beanstanden seine Kritiker / innen, dass dieser triumphalistische Optimismus durch eine größere Gewichtung der Sündhaftigkeit der Menschheit abgeschwächt werden müsse. Schließlich könne die Sünde der weltlichen Partizipation an Gott hindernd im Wege stehen. Mit anderen Worten wird Milbank vorgeworfen, verfrüht auf die eschatologische Wirklichkeit vorzugreifen, um die Vorzüge seines christlichen Sozialismus gegenüber säkularer Gesellschaftsordnungen anzupreisen.

In diesem Artikel gebe ich Antwort auf diese dogmatisch-orientierten protestantischen Kritiker/innen von Milbanks Theologie. Ich werde den bereits genannten Kritikpunkt bezüglich der Sündenlehre nuancieren, indem ich zu Milbanks Verteidigung einen Unterschied zwischen der in Christus unumstößlich erlösten Wirklichkeit und der aktiven Annahme dieser Tatsache durch die Menschheit einführe. Dass sich Milbanks Partizipationsgedanke allein auf ersteres bezieht, wird von seinen Kritiker/innen übersehen. Jedoch bemängele ich im dritten Teil dieses Artikels, dass Milbank unzureichend zwischen der vorbildhaften Akzeptanz dieser Wirklichkeit im Menschen Jesu Christo auf der einen Seite und der allgemeinen menschlichen Akzeptanz dieser erlösten Wirklichkeit auf der anderen Seite unterscheidet. Die von protestantischen Kritiker/innen beanstandete vernachlässigte Berücksichtigung der Sündenlehre trifft in diesem Zusammenhang insofern zu, als dass Milbank zu schnell von Christi Vorbild auf alle Menschen schließt. Anschließend fülle ich die Diskussion mit Einsichten aus Kathryn Tanners Theologie an, da diese imstande ist, den Nachdruck auf die Rolle der Sündenlehre seitens Milbanks Kritiker / innen mit der von mir verteidigten Version Milbanks zu vereinigen. Tanner und Milbank stimmen darin überein, dass die Erlösung der Welt in Christo als unumstößliche Wirklichkeit betrachtet werden muss und dass demzufolge menschliche Sündhaftigkeit die Partizipation der Welt an Gott nicht beeinträchtigt. Jedoch respektiert Tanner mehr als Milbank den Unterschied zwischen Jesu Christo und dem Rest der Menschheit. Dies wird verdeutlicht, indem ich derzeitige Debatten über die gegensätzliche Konzeptionalisierung des Geschenks in Milbanks und Tanners Theologien auf die Diskussion über das Verhältnis zwischen ontologischer Erlösung und anthropologischer Sündhaftigkeit beziehe. Ich argumentiere, dass Tanners Betonung des einseitig nur von Gott auf die Welt gerichteten Gnadengeschenks (entgegen der Meinung ihrer Kritiker/innen) auf einer weitaus negativeren Anthropologie basiert als Milbanks Konzeptionalisierung des Geschenks im Sinne von Austausch. Zum Abschluss erläutere ich, inwiefern Tanners Vorstellung zu einer demütigeren Positionierung des Christentums in seinem Verhältnis zur säkularen Gesellschaft führt als Milbanks Projekt. 


\section{Kritik an Milbanks Erlösungsverständnis}

Die Gesamtheit Milbanks theologischer Schriften expliziert die grundlegende These, dass die politischen Probleme säkularer Gegenwartsgesellschaften damit zusammenhängen, dass in der Moderne die Welt unabhängig von ihrer Beziehung zu Gott gedacht wurde. ${ }^{1}$ Milbank sieht diese fälschliche Trennung zwischen Gott und Welt nicht nur im Protestantismus fortgesetzt, sondern auch das säkulare Denken untermauert diese philosophische Grundannahme bis heute. Ein großer Teil von Milbanks Theologie besteht darin, die politischen Konsequenzen solch einer Ontologie zu beleuchten. ${ }^{2}$ Damit einhergehend behauptet er, dass diese säkulare Ontologie nicht nur von theologischer Seite als häretisch betrachtet, sondern auch von einer analogischen Interpretation alles weltlichen Seienden in seinem Verhältnis zu Gott ersetzt werden müsse. Gott muss erneut als die allumfassende Wirklichkeit gedacht werden, an welcher alles Weltliche partizipiert.

Während andere seine Theologie als romantisierenden Rückzug in vergangene, vorsäkulare Zeiten bezeichnen, ${ }^{3}$ wurde Milbanks Projekt, mittels klassischer theologischer Literatur neue Lösungen für Probleme westlicher Gegenwartsgesellschaften $\mathrm{zu}$ finden, seitens mehrerer gegenwärtiger protestantischer Theolog/innen lobend gewürdigt. ${ }^{4}$ Jedoch wird bemängelt, dass Milbanks Theologie zu einseitig auf katholisches Erbe und zu wenig auf protestantische Einsichten aufbaue. ${ }^{5}$ Die weitestverbreitete protestantische Kritik betrifft Milbanks bereits erläutertes Denken

1 John Milbank, Postmodern Critical Augustinianism, in Ders.: The Future of Love. Essays in Political Theology, London 2009, 351.

2 Dies ist Milbank am prägnantesten in Beyond Secular Order. Critique on Modern Ontology, Oxford 2013, gelungen.

3 Siehe z. B. Colin Gunton, Editorial, in: International Journal of Systematic Theology 1 (1999), 116. Für Milbanks Verteidigung seiner Theologie gegen Nostalgievorwürfe siehe John MilBank, Faith, Reason, and Imagination, in DeRs.: The Future of Love, 329.

4 Sven Grosse, Radical Orthodoxy - Darstellung und Würdigung einer herausfordernden Theologie, Neue Zeitschrift für Systematische Theologie und Religionsphilosophie 55 (2013), 441-442. Beispielsweise wird Milbanks Versuch begrüßt, einen christlichen Sozialismus zu konstruieren, in welchem Gerechtigkeit nicht auf Kosten einer Einebnung aller existierenden sozialen Unterschiede entsteht (ebd., 447-448). Auch die Sammlung Aufsätze über das Verhältnis zwischen Radical Orthodoxy und reformierter Theologie empfängt Milbanks Theologie (und die Theologien anderer Radical Orthodoxer Autoren) erstrangig in positivem Lichte (James K. Smith, Reverberations. Radical Orthodoxy and the Reformed Tradition, in James K. Sмiтh / James H. Olthuis (Hg.): Radical Orthodoxy and the Reformed Tradition. Creation, Covenant, and Participation, Grand Rapids, MI 2005, 15-19. Die wohl prominenteste protestantische Würdigung Radical Orthodoxys ist bei James K. Smith zu finden (James K. SмiтH, Introducing Radical Orthodoxy. Mapping a Post-secular Theology, Grand Rapids, MI, 2004).

5 Grosse (s. o. Anm. 4), 454. 
über das Verhältnis zwischen Gott und Welt. ${ }^{6}$ Es wird ihm vorgeworfen, dass er die Auswirkungen menschlicher Sündhaftigkeit auf die Beziehung zwischen Welt und Gott außer Betracht lasse. ${ }^{7}$ Die Kritiker / innen beanstanden, dass Milbank unzureichend berücksichtige, dass die scharfe Trennung zwischen Gott und Welt im Protestantismus nicht Symptom einer fälschlichen Schöpfungslehre sei. Im Gegensatz dazu ginge es der protestantischen Theologie ganz bewusst darum Gott und Welt zu trennen, da allein damit die Ernsthaftigkeit der Sünde gewürdigt werde. ${ }^{8}$ Folglich wollen die hier besprochenen Kritiker/innen die partizipatorische Beziehung zwischen Gott und Welt nicht philosophisch voraussetzen, sondern sie als eschatologisches Ziel verstehen. Nachdruck wird auf die persönliche Bekehrung zu Christo als notwendige Voraussetzung zur Erreichung dieses Ziels gelegt. ${ }^{9}$ Protestanten betonen, dass nur von und vor Gott gerechtfertigte Menschen so an Gottes Leben teilnehmen wie Milbank es darstellt. ${ }^{10}$ Der Hauptkritikpunkt ist demnach, dass Milbank illegitimerweise die Welt nach dem Sündenfall entweder mit der ursprünglichen oder mit der eschatologischen neuen Schöpfung gleichsetze.

Demzufolge würde Milbanks Kritik an der säkularen Moderne nicht zutreffen, da es Atheist / innen nicht vorgeworfen werden kann, dass sie die Welt unabhängig von ihrer Beziehung zu Gott betrachten. Von protestantischer Seite aus müsste man dementgegen eher zugeben, dass gerade dieses säkulare Denken wahrheitsgetreu die Tatsache widerspiegelt, dass Sünder/innen ihre Beziehung zu Gott verwerfen. Der Säkularimus wird dieser Interpretation zufolge einem oberflächlichen Kulturchristentum vorgezogen. Es wird als positiv bewertet, dass Menschen sich nicht mehr Christ/innen nennen, da sie eine persönliche

\footnotetext{
6 Maarten WISSE, “Pro salute nostra reparanda”. Radical Orthodoxy's Christology of Manifestation versus Augustine's Moral Christology, in NZSTh 50 (2007), 353.

7 N. Vorster, A Critical Assessment of John Milbank's Christology, in Acta Theologica 32 (2012), 294; Steven SHAKESPEARE, Radical Orthodoxy. A Critical Introduction, London 2007, 116. Es besteht jedoch auch die entgegengesetzte Kritik, Milbanks starkes Kontrastdenken zwischen Christentum und Säkularismus würde letzteren allzu sündhaft beschreiben und vergessen, dass auch dort Gottes Gnade herrsche (Elaine L. GraHAM, Between a Rock and a Hard Place. Public Theology in a Post-Secular Age, London, 2013, 107; 112-113). Auch diese Kritik wird sich als unzutreffend herausstellen, wenn ich in diesem Artikel Milbanks Verständnis der Sünde erläutere. In diesem Zusammenhang nuanciere ich auch jene, die Milbank vorwerfen, aufgrund seiner antagonistischen Position das wahrhaft Gute im modernen Säkularismus zu verkennen (Douglas HEDLEY, Review: Radical Orthodoxy. A New Theology, in: Journal of Theological Studies 51 (2000), 405-408).

8 Grosse (s. o. Anm. 4), 454; SMith, Introducing Radical Orthodoxy (s. o. Anm. 4), 122; SMIth, Reverberation (s. o. Anm.4), 20; Adrienne Dengerink Chaplin, The Invisible and the Sublime. From Participation to Reconciliation, in Smith / Olthuis (Hg.): Radical Orthodoxy and the Reformed Tradition (s. o. Anm. 4), 91; 104.
}

9 Grosse (s. o. Anm. 4), 454.

10 Grosse (s. o. Anm. 4), 458-459. 
Bekehrung zu Christo verweigern und nicht all die Konsequenzen tragen, die das wahrhafte Christsein impliziert. ${ }^{11}$ In der mittelalterlichen Reformation sei es schließlich gerade um diese Säuberung des Christentums von Scheinheiligkeit gegangen. In diesem Zusammengang wird Milbank vorgeworfen, zu einem gewissen Grad auf diesen vorreformatorischen Irrwegen fortzuschreiten, vor welchen die protestantische Theologie bereits seit Jahrhunderten warnt. ${ }^{12}$ Auch Milbank hülle allzu unvorsichtig säkulares philosophisches Gedankengut in einen theologischen Deckmantel ohne darüber nachzudenken, inwiefern eine persönliche Bekehrung zu Christo diese rein philosophische Übersetzung beeinflussen müsste. ${ }^{13}$ Um im ausgeführten Sinne nicht alleine oberflächlich christlich und demzufolge fundamental unchristlich zu werden, müsse Milbank das Gute, was die Reformation zum Christentum beigetragen habe, mehr wertschätzen.

Nach dieser kurzen Darstellung der Diskrepanz zwischen Milbanks Position und seinen protestantischen Kritiker/innen werde ich im Folgenden argumentieren, dass John Milbank sehr wohl den Einfluss der Sünde auf die Beziehung der Welt zu Gott berücksichtigt. Im Anschluss an diese Verteidigung wird jedoch auf den Kritikpunkt zurückgegriffen werden, Milbank hebe die einzigartige Rolle Christi in der weltlichen Partizipation an Gott unzureichend hervor. ${ }^{14}$ Mit anderen Worten müsste in der Tat die fortwährende Abhängigkeit der Menschen von Christo eine zentralere Stelle einnehmen als Milbank ihr dies zugesteht.

\section{Erlösung als positiver Exzess der Gnade Gottes}

Um Milbanks Position besser verstehen zu können, muss hervorgehoben werden, dass die Sündenlehre zwei Problematiken beinhaltet. Das Problem der Sünde

11 Grosse (s. o. Anm.4), 458-459; Maarten WISSE, «...welches alle Menschen erleuchtet?». Die Krise der europäischen Identität im Spiegel der frühmodernen Rezeption des Johannesprologs, in: NZSTh 55 (2013), 16.

12 Grosse (s. o. Anm. 4), 461. Auch Wisse behauptet, dass nur vor der Reformation die Erlösung universell von der katholischen Kirche versprochen wurde und an äußeren Lebensweisen und Ritualen erkennbar war, wer zu den Erlösten zählt. Mit der Reformation sei es unsicherer geworden, wer erlöst ist (WISSE, «...welches alle Menschen erleuchtet?» (s. o. Anm. 11), 4).

13 Grosse (s. o. Anm.4), 458-459. Diese Aufgabe der theologischen Integrität zu Gunsten der Philosophie wurde auch von anderen beanstandet (Todd J. Billings, John Milbank's Theology of the „Gift“ and Calvin's Theology of Grace. A Critical Comparison, in: Modern Theology 21 (2005), 87-105; Graham (s. o. Anm. 7), 135).

14 Für eine Kritik, dass Milbank gerade mehr die Göttlichkeit als die Menschlichkeit Jesu Christi betone, siehe Frederick Christian Bauerschmidt, The Word Made Speculative? John Milbank's Christological Poetics, in: Modern Theology 15 (1999), 417-432. 
wirft einerseits die Frage nach Gottes Beziehung zur Welt, und zum Anderen nach der Beziehung der Welt zu Gott auf. Dies bedeutet, dass zum Einen beantwortet werden muss, inwiefern die Erbsünde Gottes Beziehung zur Welt schädigt und zum Anderen, wie die Erbsünde die Beziehung der Welt zu Gott beeinträchtigt. Im Folgenden erläutere ich, dass Milbank auf beide Fragen mit Gottes Gnade antwortet, welche er sich als Selbst-Exzess vorstellt. ${ }^{15}$ Zusammenfassend beinhaltet dieses Gnadenverständnis, dass der Sünde kein Einfluss auf Gottes Beziehung zur Welt zugestanden wird. ${ }^{16}$ Jedoch bejaht Milbank den Einfluss der Sünde auf die Beziehung der Welt zu Gott. ${ }^{17}$ Im folgenden erkläre ich Milbanks Positionierung zu beiden Seiten der Sündenproblematik ausführlicher.

Der augustinischen Tradition folgend behauptet Milbank, dass die Schöpfung auch bevor dem Sündenfall allein durch Gottes fortwährendes Gnadenangebot gut war. ${ }^{18}$ Dass dieses Angebot auch nach dem Sündenfall noch besteht, ist für Milbank keine Selbstverständlichkeit, sondern genau diese Einsicht wurde in Jesu Christo bewirkt und sichtbar. In Christo wurde die mysteriöse - im Sinne von unberechenbare - Gutheit Gottes offenbart. ${ }^{19}$ An Christum zu glauben bedeutet demzufolge zu vertrauen, dass die Unerschöpflichkeit der Gnade Gottes auch nach dem Sündenfall die gesamte Wirklichkeit durchströmt. ${ }^{20}$ Denn diese Gnade existiert immer noch unbeeinträchtigt in all ihrer Perfektion in Gott selbst. Gerade in Christo wurde offenbar, dass Gott auf die menschliche Ablehnung des unendlichen Gnadenangebots Gottes nicht mit dem berechenbaren Rückzug dieser Gnade reagiert. Anstelle dieser kalkulierbaren Reaktion bietet Gott der Welt ein erneutes Gnadengeschenk an, welches das ursprüngliche Schöpfungsgeschenk sogar noch übertrifft. Dies bedeutet nicht, dass Milbank Gottes unbeeinträchtigtes Gnadenangebot an die Welt trotz des Sündenfalls verteidigt, weil er die Sünde nicht ernst nähme. Im Gegenteil: er unterstreicht den Exzess göttlicher Gnade über alles menschliche Fehlen.

15 John Milbank, Theology and Social Theory. Beyond Secular Reason, Oxford 1990,2006, 12. Da Milbank Gott in sich selbst als positiven Exzess versteht, interpretiert er auch Gottes Unbegreiflichkeit nicht in erster Linie epistemologisch, sondern ontologisch: Gottes eigenes Wesen überschreitet stets das, was Menschen zu einem gewissen Zeitpunkt begreifen können (John Milbank, The Force of Identity, in DERS.: The Word Made Strange: Theology, Language, Culture, Oxford 1997, 201).

16 Milbank, The Second Difference, in Ders.: The Word Made Strange (s. o. Anm. 15), 182-183.

17 Milbank, Postmodern Critical Augustinianism (s. o. Anm. 1), 344.

18 John Milbank, Foreword, in Smith: Introducing Radical Orthodoxy (s. o. Anm. 4), 17.

19 John Milbank, Forgiveness: The double waters, in DeRs.: Being Reconciled: Ontology and Pardon, Oxon 2004, 46-47.

20 Milbank, The Second Difference, in Ders.: The Word Made Strange (s. o. Anm.15), 182-183. 
In diesem Zusammenhang muss auch Milbanks Behauptung verstanden werden, dass eine christozentrische Ontologie die Wirklichkeit von der Perspektive der Erlösung und nicht von der Perspektive des Bösen her interpretieren muss. ${ }^{21}$ Schließlich ist die ganze Welt bereits erlöst, insofern sie all ihre Gutheit noch stets von Gottes ununterbrochenem Gnadengeschenk bezieht. Alles Sündhafte und Böse stellt lediglich ein zweitrangiges Eindringen in diese grundsätzliche Erlösungswirklichkeit dar. ${ }^{22}$ In diesem Sinne hat sich an Gottes Beziehung zur Welt durch den Sündenfall nichts verändert. ${ }^{23}$ Die gesamte erschaffene Wirklichkeit bleibt von Gottes Liebe durchströmt. Jedoch ist dieser unveränderte Zustand in der Zeit nach dem Sündenfall vollkommen überraschend und neu.

Diese Vorstellung, dass Gottes Perfektion vom Sündenfall unbeeinträchtigt bleibt, wurde von protestantischer Seite hinsichtlich der Frage um Gottes Gerechtigkeit kritisiert. ${ }^{24}$ Hier wird der Gedanke der Buße angeführt um zu gewährleisten, dass Gottes Erbarmen nicht auf Kosten von Gottes Gerechtigkeit hervorgehoben wird. Dieser Argumentation liegt die Behauptung zugrunde, dass Gott ungerecht wäre, wenn seine Gnade gleichermaßen an Sünder / innen wie an bußfertige Menschen verteilt würde. Milbank hingegen unterstreicht, dass Gottes Vergebung die menschliche Buße immer schon beinhaltet. ${ }^{25}$ Seine Auffassung von Gottes Wesen als positiver Exzess beinhaltet, dass Gottes Vergebung nie allein das negative Auslöschen einer Sünde ist, sondern neue Positivität erschafft. Diese ist sichtbar in der menschlichen Buße vermittelt. Jedoch hängt dies nicht erstrangig mit Gottes Gerechtigkeit zusammen, denn die Gnade ist in jedem Fall gleichermaßen unverdient. ${ }^{26}$ Für Milbank ist die Buße gerade deshalb ein integraler Bestandteil weltlicher Vermittlung der Gnade Gottes, weil Gott nach dem Sündenfall in sich perfekt, das will heißen positiver Exzess, bleibt. Es ist dann nicht der Fall, dass Gott nicht-bußfertigen Menschen nicht vergeben will. Vielmehr manifestieren die nicht-Bußfertigen in ihrem sich Verschließen vor Versöhnung, dass sie Gottes Gnadenangebot immer noch ablehnen.

Was die Frage der Auswirkungen der Erbsünde auf die Beziehung der Welt zu Gott betrifft, argumentiert Milbank hier demnach für einen von seinen Kritiker/innen unbeachteten Bruch mit der ursprünglichen Schöpfungsordnung. Milbank definiert Sünde als die Abkehr von Gottes Liebe. ${ }^{27}$ Aufgrund ihrer Abkehr

21 Milbank, Introduction, in Ders.: Being Reconciled (s. o. Anm. 19), xii.

22 Milbank, The Poverty of Niebuhrianism, in Ders.: The Word Made Strange (s. o. Anm. 15), 244.

23 Milbank, The Second Difference, in Ders.: The Word Made Strange (s. o. Anm. 15), 182-183.

24 WISSE, "Pro salute nostra reparanda" (s. o. Anm. 6), 370.

25 Milbank, Forgiveness, in Ders.: Being Reconciled (s. o. Anm. 19), 45-46.

26 Milbank, Forgiveness, in Ders.: Being Reconciled (s. o. Anm. 19) , 44.

27 Milbank, Postmodern Critical Augustinianism (s. o. Anm. 1), 344. 
von Gottes Liebe ist die sündhafte Menschheit nicht länger fähig, die Erlösung der Wirklichkeit zu erkennen. Demzufolge ist auch die Kritik unberechtigt, Milbanks These der Allgegenwärtigkeit von Gottes Gnade unterminiere jedweden Einfluss der Sünde auf das menschliche Erkenntnisvermögen. ${ }^{28}$ Entgegen diesem Vorwurf geht Milbank gerade nicht von einer allgemeinen Erleuchtung des menschlichen Verstandes aus, wie ihm vorgeworfen wird. Milbank argumentiert vielmehr, dass die christliche Offenbarung den menschlichen Verstand auf die bereits erleuchtete Wirklichkeit, die ihn umgibt, richten muss. Eine der fundamentalsten Sünden in dieser Situation ist es, die Abhängigkeit der Welt von Gottes Gutheit zu leugnen und davon auszugehen, dass die Welt selbstständig und autark durch die freie Wahl der Menschen gut sein kann. ${ }^{29}$ In solch einer Haltung manifestiert sich die Sünde des Stolzes.

Von diesem Hintergrund aus betrachtet kann nun der oben genannte protestantische Einwurf, dass die mittels menschlicher Kräfte unüberbrückbare Kluft nicht zwischen dem unendlichen Gott und endlichen Geschöpfen, sondern zwischen dem perfekten Gott und der sündhaften Welt bestände, ${ }^{30}$ nuanciert werden. Es muss hervorgehoben werden, dass auch für Milbank die Sünde die entscheidende Kluft zwischen Welt und Gott entstehen lässt, welche es durch die Erlösung zu überwinden gilt. Jedoch argumentiert er, dass die Sünde nur eine Kluft von menschlicher Seite aus darstellt, wohingegen Gottes Beziehung zur Welt trotz der Sünde ungetrübt bleibt. Der Exzess des Gnadenangebots Gottes bedeutet im Hinblick auf die menschliche Sündhaftigkeit, dass Gottes Gnade nach dem Sündenfall nicht mehr allein im fortwährenden Angebot der Gutheit Gottes an die Welt besteht, sondern darüber hinaus nun auch für die Heilung der menschlichen Natur bereitsteht. ${ }^{31}$ Ich stimme Milbank also zu, dass er entgegen der Behauptung seiner Kritiker/innen mit einer überaus robusten Sündenlehre arbeite. ${ }^{32} \mathrm{Ihm}$ zufolge durchzieht die Sünde die Schöpfung in einer Weise, dass die Welt nun gleichzeitig perfekt und schlecht ist. In ihrer erlösten und damit absoluten Wirklichkeit ist die Welt perfekt, doch in ihrer historischen Sündhaftigkeit ist die Welt noch schlecht. Dass die Wirklichkeit auch nach der Sünde noch stets allein von Gottes Gutheit durchströmt ist, ist kein selbstverständlicher Automatismus, der in Christo lediglich sichtbar wurde,

28 Smith, Introducing Radical Orthodoxy (s. o. Anm. 4), 165.

29 Milbank, 'Evil. Darkness and Silence', in Ders.: Being Reconciled (s. o. Anm.19), 8. Siehe auch Milbank, 'Forgiveness', in Ders.: Being Reconciled (s. o. Anm.19), 46-47 und Milbank, Postmodern Critical Augustinianism (s. o. Anm.1), 344.

30 Grosse (s. o. Anm. 4), 456.

31 Milbank, Foreword, in Smith: Introducing Radical Orthodoxy (s. o. Anm. 4), 17.

32 Milbank, Foreword, in Smith: Introducing Radical Orthodoxy (s. o. Anm. 4), 17. 
sondern in seiner Kontinuität ist Gottes Gnadenangebot nach dem Sündenfall vollkommen neu.

In Verteidigung gegen die Vorwürfe seiner Kritiker / innen muss also festgehalten werden, dass Milbanks Optimismus hinsichtlich einer partizipatorischen Interpretation der Beziehung zwischen Gott und Welt auch nach dem Sündenfall nicht auf Kosten einer Vernachlässigung der Sündenlehre erworben wurde. Im Gegenteil, Milbank argumentiert gerade aus der Sicht von Gottes fortwährendem Erlösungsangebot an die Welt. Um gut zu sein, bleibt die Welt nach dem Sündenfall wie auch vor diesem in einer radikalen Abhängigkeitsbeziehung zu Gott. Demzufolge setzt Milbank nicht voraus, dass jeder Mensch Gottes Gnadenangebot adäquat annimmt und in eine persönliche Beziehung zu Gott eintritt. Er betont schlechthin, dass diejenigen, welche sündhaft bleiben, das Wesen Gottes (als positiven Exzess) damit nicht verändern. Im Gegensatz zu den Sündhaften geben jene, welche Gottesgnadenangebot annehmen, ein Gegengeschenk an Gott. Dies muss so verstanden werden, dass jeder erlöste Mensch einen Exzess zur bereits bestehenden Gutheit der erlösten Wirklichkeit darstellt. ${ }^{33}$ Die erlöste Wirklichkeit nimmt durch jeden neuen erlösten Menschen an Gutheit zu. Die Erlösten partizipieren also auch aktiv an Gottes Wesen und sind nicht nur passive Nutznießer.

Dies hängt damit zusammen, dass Milbank die Überwindung der Sünde durch die Gnade nicht lediglich als Behebung eines Mangels ansieht. ${ }^{34}$ Wie sich bereits an Milbanks Bußverständnis ablesen lässt, behauptet er, dass die Struktur der Gnade davon gekennzeichnet ist, dass das Ergebnis stets die Veranlassung oder den zu überwindenden Mangel übersteigt. ${ }^{35}$ Dies leitet er unmittelbar von der Inkarnation Christi ab. In Christo wurde das verlorene Gut der ursprünglichen Schöpfung nicht nur wiederhergestellt, sondern die Inkarnation Christi übertrifft die ursprüngliche Schöpfung in ihrer Gutheit. Davon leitet Milbank ab, dass in jedem Fall, wenn das Böse durch Gnade erlöst wird, das ehemals Sündhafte nun in seiner Positivität wiederholt wird. ${ }^{36}$ Jedes Mal, wenn ein Mensch Gottes Erlösungsangebot aktiv annimmt, versiegt damit die sündhafte Vergangenheit nicht nur im Nichts, sondern es wird zudem ein neues, noch nie dagewesenes Gut durch dieses erlösende Überkommen der Sünde erschaffen. ${ }^{37}$

33 John Milbank, The Programme of Radical Orthodoxy, in Laurence Paul Hemming (Hg.), Radical Orthodoxy? - A Catholic Enquiry, Aldershot 2000,44; Milbank, Incarnation. The Sovereign Victim, in DeRs.: Being Reconciled (s. o. Anm. 19), 66-70.

34 Milbank, Incarnation, in Ders.: Being Reconciled (s. o. Anm.19), 67.

35 Milbank, The Programme of Radical Orthodoxy, (s. o. Anm. 33), 44; Milbank, Incarnation, in DERS.: Being Reconciled (s. o. Anm. 19), 66-70.

36 Milbank, The Name of Jesus, in Ders.: The Word Made Strange (s. o. Anm.15), 155.

37 Milbank, The Name of Jesus, in Ders.: The Word Made Strange (s. o. Anm. 15), 155. 


\section{Der Unterschied zwischen Christo und der erlösten Menschheit}

Genau diese letzte Gedankenbewegung will ich im Folgenden nuancieren. Meine Kritik lehnt an die oben angeführte Bemerkung an, Milbank unterminiere in seiner Ontologie die Notwendigkeit einer persönlichen Bekehrung zu Christo. ${ }^{38}$ Obwohl hinreichend ausgeführt wurde, dass diese persönliche Bekehrung nicht für die Wirklichkeit der ungetrübten Gottes-Welt-Beziehung notwendig ist, entnehme ich diesen Kritiker / innen, dass stärker zwischen Jesu Christo und dem Rest der Menschheit unterschieden werden muss als Milbank dies zulässt. Zunächst will ich jedoch darauf hinweisen, dass ich mich von den Kritiker / innen unterscheide, welche bemängeln, Milbank fasse die Inkarnation nicht im Sinne einer grundsätzlichen Veränderung, sondern nur im Sinne einer Wiederholung der ursprünglichen Gottesoffenbarung an die Schöpfung auf. ${ }^{39}$ Wie bereits erläutert, argumentiert Milbank nachvollziehbar dafür, dass nach dem Sündenfall das fortwährende Gnadengeschenk Gottes in Christo völlig neu und unerwartet war. Auch streite ich ab, dass das Problem mit Milbanks angeblich inkarnatorischem Erlösungsverständnis zusammenhängt. ${ }^{40}$ Dies würde bedeuten, dass bereits mit der Menschwerdung Gottes (und nicht etwa erst durch den Kreuzestod und die Auferstehung) die gesamte Welt als bereits erlöste offenbart wurde. Schließlich unterstreicht Milbank sowohl, dass Christus die Welt bereits vollkommen erlöst hat, als auch, dass jeder Mensch diese Erlösung mit dem eigenen Leben nachvollziehen muss. ${ }^{41}$

Jedoch stimme ich den Kritiker / innen zu einem gewissen Grad darin zu, dass Milbank die Einzigartigkeit Christi bagatellisiert. ${ }^{42}$ Meiner Interpretation zufolge

38 Hier wird Milbank zwar beigepflichtet, dass sich vom christlichen Glauben ontologische Aussagen über die gesamte Wirklichkeit ableiten lassen, jedoch wird bemängelt, dass Milbank den Akzent dermaßen auf die Ontologie setze, dass er den für das Christentum fundamentalen Bezug zur Bibel und zur partikulären Heilsnotwendigkeit Christi übermale (GRossE (s. o. Anm.4, 437). Zur Radical Orthodoxen Vernachlässigung der Bibel und christlicher Praxen zugunsten philosophischer Kohärenz siehe auch Laurence Paul Hemming, Analogia non Entis sed Entitatis. The Ontological Consequences of the Doctrine of Analogy, in: International Journal of Systematic Theology 6 (2004), 119-120, 129; David FoRD, 'Radical Orthodoxy and the Future of British Theology', in: Scottish Journal of Theology 54 (2001), 394, 397-398; Graeme RICHARDson, Integrity and Realism. Assessing John Milbank's Theology, in: New Blackfriars 84 (2007), 273; R. R. Reno, The Radical Orthodoxy Project, in: First Things, February 2000, http://www. firstthings.com/article/2000/02/the-radical-orthodoxy-project) (accessed: 08. 06. 2014).

39 Wisse, «...welches alle Menschen erleuchtet?» (s. o. Anm.11), 8.

40 Wisse, «...welches alle Menschen erleuchtet?» (s. o. Anm.11), 9.

41 Milbank, Postmodern Critical Augustinianism (s. o. Anm.1), 345.

42 WisSE, «...welches alle Menschen erleuchtet?» (s. o. Anm.11), 9. 
besteht Milbanks Fehler darin, die Struktur von Christi beispielhafter Akzeptanz des Gnadenangebots Gottes direkt auf die Akzeptanz dieses Gnadengeschenks aller erlösten Menschen zu übertragen. Ich gebe Milbank zwar Recht, dass Christi exemplarische aktive Annahme der Gnade Gottes mehr als nur die Behebung eines Mangels war. Jesus Christus heilte die Welt nicht nur von der Sünde, sondern verkörperte darüber hinaus auch ein vollkommen neues Gnadengeschenk an die Welt. Jedoch behaupte ich, dass im Falle erlöster Menschen diese Struktur des Ergebnisses, welches seinen Anlass an Positivität übersteigt, stets von der Sünde beeinträchtigt wird. Dies bewirkt, dass das Wachstum der Welt an Gutheit mehr kreuzförmig als triumphierend ist. Um mein Argument zu untermauern, wende ich mich nun an Einsichten der US-amerikanischen protestantischen Gegenwartstheologin Kathryn Tanner.

Ebenso wie John Milbank argumentiert auch Kathryn Tanner für eine ungebrochene Beziehung Gottes zur Welt trotz des Sündenfalls. ${ }^{43}$ Auch Tanner beschreibt Gnade als Gottes fortwährendes Angebot wahrer Gutheit an die Welt ungeachtet des Sündenfalls. Jedoch unterscheidet sich Tanners Verständnis des Gnadengeschenks von Milbanks darin, dass Tanner das Geschenk als fundamental einseitig von Gott auf die Welt gerichtet betrachtet. Milbank dagegen versteht das Geschenk im Sinne des reziproken Austauschs. ${ }^{44}$ Zunächst einmal muss festgehalten werden, dass im Zusammenhang dieses Artikels es gerade Milbanks Schwerpunktsetzung auf den Austausch ist, welche seinen Fokus so vehement auf das Wachstum des wirklich Guten durch jeden erlösten Menschen lenkt. Wie bereits erläutert versteht Milbank die aktive Akzeptanz Gottes Gnadengeschenks von menschlicher Seite als Gegengeschenk der Menschheit an Gott. Milbank spricht von einem paradoxen unilateralen Austausch, insofern das menschliche Gegengeschenk an Gott nicht selbstständig ist, sondern immer noch auf fundamentalster Ebene von Gott gegeben ist. ${ }^{45}$ Das Wachstum der Erlösungswirklichkeit basiert jedoch auf solch einem asymmetrischen Austausch zwischen Gott und Welt. Im letzten Teil dieses Artikels entgegne ich nun Milbank, dass

43 Kathryn, Tanner, Christ the Key, Cambridge 2010), 134.

$44 \mathrm{Zu}$ diesem Vergleich siehe David ALBERTSON, On “The Gift” in Tanner's Theology. A Patristic Parable, in: Modern Theology 21 (2005), 107-118. Siehe ebenfalls Sarah CoAKLEY, Gift, gender and Trinitarian relations in Milbank and Tanner, in: Scottish Journal of Theology 61 (2008), 224235. Für eine Kritik, dass sich Milbanks und Tanners Verständnis des Geschenks weniger unterscheiden als Tanner es behauptet, siehe Daniel BeLL, Review: Economy of Grace, in: Reviews in Religion and Theology 14:2 (2007), 235-237. Für eine Kritik, welche Tanners Position derjenigen Milbanks bevorzieht, siehe Jan-Olaf HenRIKSEn, Desire, Gift and Recognition. Christology and Postmodern Philosophy, Grand Rapids, Michigan / Cambridge 2009).

45 John Milbank, The Suspended Middle. Henri de Lubac and the Debate concerning the Supernatural, London 2005, 91. 
das Gnadengeschenk Gottes an die Menschheit nur in Jesu Christo und einigen besonders exzellenten menschlichen Fällen im Sinne von Austausch verstanden werden kann. In den meisten Fällen ist das Geschenk jedoch einseitig von Gott an die Welt gerichtet.

Dazu bediene ich mich eines für diese Argumentation wichtigen Unterschieds zwischen einer optimistischen Ontologie und einer pessimistischen Anthropologie, der in Kathryn Tanners Theologie zum Vorschein tritt. Zunächst ist es beachtlich, dass Tanner, gleichsam John Milbank, regelmäßig für die NichtBerücksichtigung der Sündenlehre in ihrer Theologie kritisiert wird. ${ }^{46}$ Ebenso wurde Tanner, gleichsam John Milbank, dafür kritisiert, die Inkarnation nicht als Gottes Antwort auf die Sünde, sondern lediglich als sichtbare Manifestation Gottes ewigen Gnadenangebots darzustellen. ${ }^{47}$

Beide Kritikpunkte sind unberechtigt, wenn bedacht wird, dass Tanners Anthropologie aufgrund ihres Sündenverständnisses pessimistischer ist, als ihre Kritiker / innen erkennen und zugeben. Tanner zufolge impliziert die menschliche Ablehnung der Gnade Gottes, dass die menschliche Natur ihre ursprüngliche Gutheit verliert, in der sie geschaffen wurde. ${ }^{48}$ Dies bedeutet, dass einerseits die Wirklichkeit durch die Erlösung fundamental gut, andererseits jedoch die menschliche Vermittlung dieser Gutheit problematisch ist. Die menschliche Akzeptanz der Gnade Gottes bleibt fehlerhaft und unvollkommen. Dies bedeutet, dass die aktive menschliche Annahme der Gnade Gottes nicht in jedem Fall ein Gegengeschenk an Gott darstellt. Die menschliche Vermittlung entstellt Gottes Gnade öfter, als dass sie zum Wachstum im wahrhaft Guten beitragen würde. ${ }^{49}$ Laut Tanner geben Menschen immer in verzerrter und niedrigerer Form das zurück, was sie von Gottes Gnade empfangen haben. Das Gute wird dadurch fortwährend durch die menschliche Sündhaftigkeit beschädigt. ${ }^{50}$

Wohingegen Milbank vorranging am Wachstum des Guten interessiert ist, legt Tanner somit den Nachdruck auf die Wiederherstellung der ursprünglichen Gutheit. Was Gott an der Erlösung einzelner Menschen gewinnt, ist für sie nur von zweitrangiger Bedeutung. ${ }^{51}$ Empfangene Gnade ist kein Gegengeschenk an

46 Albertson (s. o. Anm. 44), 108; Bell (s. o. Anm. 44) , 236-237; Chris W. Hackett, Review Essay. What's the Use of a Skeleton Key for Christian Theology? A Report on an Essential Problematic in Kathryn Tanner's Christ the Key, in: Radical Orthodoxy: Theology, Philosophy, Politics 2 (2014), 209; David A. Ford, Review: God and Creation in Christian Theology. Tyranny or Empowerment? by Kathryn Tanner, in: Religious Studies 26 (1990), 552.

47 Oliver CRISP, Revisioning Christology. Theology in the Reformed Tradition, Farnham 2011, 117.

48 Tanner, Christ the Key, 34-35.

49 TANner, Christ the Key, 66-67.

50 TANnER, Christ the Key, 68.

51 Kathryn TANNER, Economy of Grace, Minneapolis 2005, 68-69; 71. 
Gott, sondern sie wird zugleich an andere Geschöpfe verteilt. ${ }^{52}$ Damit schließt Tanner nicht aus, dass die Wirklichkeit noch an Gutheit zunehmen kann oder, mit anderen Worten, dass Menschen ein Gegengeschenk an Gott leisten könnten. ${ }^{53}$ Jedoch unterstreicht Tanner mehr als Milbank, dass solch ein Gegengeschenk nur die überraschende und außergewöhnliche Ausnahme im Vergleich zum Normalfall darstellt.

Damit hebt Tanner mehr als Milbank hervor, dass Gottes fortwährendes Gnadenangebot trotz menschlicher Sündhaftigkeit an sich immer schon der Exzess der Gutheit Gottes über alles Böse ist. Auch mit Tanner lässt sich Gottes überfließende Gutheit im Sinne eines wachsenden Überschusses vorstellen. Indem sie jedoch zwischen der sündlosen Natur Christi und der sündhaften Natur der restlichen Menschheit unterscheidet, wird es ermöglicht, sich den Exzess an Positivität weniger triumphal und mehr kreuzförmig vorzustellen. In den meisten Fällen, wenn Menschen auf die Gutheit der Wirklichkeit reagieren, ist dies keine wirkliche Erweiterung der Gutheit, sondern eine Verfälschung derselben. Dennoch lässt diese Verfälschung die Wirklichkeit des Guten intakt und gerade darin besteht der Exzess des wirklich Guten. Wohingegen Milbank argumentiert, dass das wahrhaft Gute sich ständig übersteigen muss, um Gottes Natur als positivem Selbstexzess treu zu bleiben, sensibilisiert uns Tanner dafür, dass selbst wenn kein einziger Mensch adäquat auf die wirkliche Gutheit Gottes reagiert, diese Gutheit dennoch vollkommen bleibt. In diesem Falle manifestiert sich der Selbstexzess historisch in der fortwährenden Gutheit der Wirklichkeit trotz aller menschlicher Verfälschung.

\section{Schluss}

Ziel dieses Artikels war es, die Bedeutung christlicher Dogmen für das Verständnis der Beziehung zwischen Christentum und säkularen Gegenwartsgesellschaften zu erkunden. Dazu habe ich die christliche Überzeugung untersucht, dass die gesamte Wirklichkeit in Christo bereits erlöst ist. John Milbank wurde als einer der gegenwärtig am häufigsten diskutierten Vertreter der These, dass die Partizipation der Welt an Gott universell wahr ist, vorgestellt. Diese These wurde gegen die Kritik verteidigt, dass sie die Sündhaftigkeit der Menschheit unzureichend berücksichtige. Es wurde gezeigt, dass in Christo gerade Gottes fortwährendes Gnadenangebot an die Menschheit trotz

52 TANNER, Economy of Grace, 70.

53 TANNER, Economy of Grace, 71-72. 
der Erbsünde offenbart wurde. Demzufolge partizipieren auch diejenigen, welche dieses Gnadenangebot ablehnen, wahrhaft an Gott. Partizipation muss in diesem Zusammenhang als die uneingeschränkte Abhängigkeit weltlicher Gutheit von Gott aufgefasst werden.

Jedoch habe ich mich in diesem Artikel kritisch mit Milbanks Behauptung auseinandergesetzt, dass erlöste Menschen nicht nur passiv von Gottes Gnade abhängig sind, sondern diese darüber hinaus auch aktiv erweitern. Durch die Erlösten wächst die Welt an Gutheit und partizipiert somit nicht nur passiv, sondern auch aktiv an Gott. Zur Nuancierung dieser Position wurde Kathryn Tanners Theologie herangezogen, welche mit Milbank hinsichtlich der Frage der ungebrochenen Partizipation der Welt auch nach dem Sündenfall an Gott zustimmt. Im Gegensatz zu Milbank ermöglicht Tanner es uns besser anzuerkennen, dass auch die Erlösten noch sündhaft bleiben. Allein Christus und manch außergewöhnliche Erlöste nehmen Gottes Gnade so an, dass sie damit aktiv an Gottes selbstübersteigender Gutheit partizipieren. Die meisten Erlösten jedoch sind lediglich passive Empfänger dieses Exzesses in dem Sinne, dass ihr verfälschtes Gegengeschenk an Gott der wirklichen Partizipation der Welt an Gott nicht schadet.

Für die Diskussion bezüglich der Beziehung zwischen Christentum und säkularen Gegenwartsgesellschaften bedeutet dies, dass sowohl Milbank als auch Tanner zufolge die säkulare Moderne zu einem gewissen Grad als sündhafte Ablehnung des Gnadengeschenks Gottes bewertet werden kann. Beide würden jedoch bejahen, dass säkulare Atheist/innen dennoch an Gott partizipieren. Sie sind immer noch von der Gutheit der Wirklichkeit abhängig und sie können immer noch in jedem Moment diese Gutheit aktiv in Empfang nehmen. Milbank preist nun die Superiorität seiner christlichen Ontologie über ihr säkulares Gegenstück an, da er davon ausgeht, dass ein größeres Gut entstehen wird, wenn Menschen die fundamentale Wirklichkeit der Erlösung erkennen und annehmen. Eine Kultur, welche Gottes Gnadenangebot sieht, ist besser als eine Kultur, welche die Erlösungswirklichkeit nicht erfassen zu vermag. Wenn wir nun Milbanks Vorstellung folgen würden, müsste jede christliche Beschäftigung mit säkularen Gegenwartsgesellschaften auf dem Glauben gründen, dass säkulare Atheist / innen immer noch fundamental auf die Wirklichkeit der Erlösung gerichtet sind. Ihre sündhafte Ablehnung der Erlösung dringt verfälschend in Gottes Beziehung zu ihnen ein, jedoch wird diese Beziehung dadurch nicht aufgelöst. Das Christentum müsste sich demnach nicht auf die Verhinderung dieser Abkehr vom Guten, sondern auf das Potenzial im säkularen Atheismus zum erweiterten Wachstum im Guten der Gesamtgesellschaft konzentrieren. Der Nachdruck müsste auf die speziellen positiven Charakteristiken säkularer Gegenwartsgesellschaften gelegt werden und politische Projekte müssten 
darauf zielen, diese zu verbessern..$^{54}$ Milbanks Verständnis zufolge würde die Sündhaftigkeit der Vergangenheit damit überwunden sein und ein noch nicht vorhandenes Gut würde entstehen. Das Ergebnis der christlichen Begegnung mit säkularen Gegenwartsgesellschaften hätte somit die Veranlassung - nämlich die Korrektur häretischen Denkens - überstiegen.

Würden wir Kathryn Tanners Theologie folgen, müssten wir zunächst einmal zugeben, dass Christ/innen, obgleich sie die Wirklichkeit der Erlösung überall sehen und bejahen, dennoch in den meisten Fällen diese Wirklichkeit nur unzureichend $\mathrm{zu}$ vermitteln imstande sind. Demnach kann eine christliche Kultur nicht versprechen besser zu sein als ihr säkulares Gegenstück. Die Begegnung mit säkularen Gegenwartsgesellschaften würde dann nicht darauf zielen ein Ergebnis zu erreichen, welches den Anlass übersteigt. Das erstrangige Ziel wäre nicht, ein wirkliches Wachstum des Guten zu fördern. Anstelle dessen würde zunächst die Beständigkeit des Guten trotz der Abkehr und Verfälschung dieses Guten durch säkulare Atheist / innen sowie auch Christ/innen gefeiert werden. Christ/innen und säkulare Atheist / innen müssten darauf aufmerksam gemacht werden, dass die Wirklichkeit trotz aller menschlicher Sündhaftigkeit fundamental gut bleibt. Christ / innen müssten nicht so sehr nach positiven Charakteristika im säkularen Atheismus als nach dem Guten in ihrer Umwelt, welches vergangene Fehler überstanden hat, suchen. Das Augenmerk der gesamten Gesellschaft müsste dann auf dieses unbesiegbare Gute gerichtet werden. Wiederum würden Christ / innen und Atheist / innen dieses Gute in verfälschender Weise empfangen und weitergeben und nicht ihr volles Potenzial für Wachstum im Guten aktivieren. Und gerade darin wäre der Glaube bestätigt, dass Gott fortwährend neue Güter an die Welt schenkt, unbeachtet aller menschlicher Beschädigung derselben.

$54 \mathrm{Zu}$ Milbanks Ablehnung von rein auf die Schadensbegrenzung orientierten negativen Aufklärungsethiken und seinem Plädoyer für eine radikale ethische Orientierung am Wachstum im Guten, siehe Milbank, Can Morality Be Christian?, in Ders.: The Word Made Strange (s. o. Anm.15), 219-224 sowie Milbank, Grace: The midwinter sacrifice, in DeRs.: Being Reconciled (s. o. Anm.19), 150. 